\title{
Measurement Invariance and Psychometric Properties of the Reactive and Proactive Aggression Questionnaire (RPQ) Across Genders
}

\begin{abstract}
Aggression is an important risk factor that predisposes adolescents to disruptive and criminal behaviours. The purpose of the present study was to investigate the structural invariance and psychometric properties of the Persian version of the Reactive-Proactive Aggression Questionnaire (RPQ) across genders among adolescents. A sample of 450 students $\left(\mathrm{M}_{\mathrm{age}}=14\right.$ years, $\left.\mathrm{SD}=0.81\right)$ was recruited randomly and completed the Persian version of the RPQ, Child Behavior Checklist and Strengths and Difficulties Questionnaire. Although the two-factor model obtained the best fit across genders, the results did not support the equivalence of factor loadings across groups. These findings replicated the distinction of reactive and proactive aggression while implied different models of the RPQ based on gender. Suggestions for future research and a more accurate assessment of these two kinds of aggression are further discussed.

Keywords: Reactive Aggression, Proactive Aggression, Adolescents, Gender, Invariance
\end{abstract}




\section{Introduction}

Research suggests that aggression is a severe and pervasive problem in adolescence, contributing to most crimes (Bobadilla et al., 2012; Cima \& Raine, 2009; Justice \& Justice, 1999; Vitaro et al., 2006). It is defined as behaviours aimed to harm people and/or objects

(Dodge, 1991). Besides the different forms of aggression, it is believed that aggression has different functions (Hubbard et al., 2010; Kempes et al., 2005). Therefore, different categories of aggression have been emerged to explain this heterogeneous structure (Cenkseven-Önder et al., 2016).

A frequently-used dichotomy classification was first put forwarded by Dodge and Coie (1987), resulting in two subtypes of aggression, proactive and reactive (Cima \& Raine, 2009; Conaty, 2006). Reactive aggression is defined by impulsive behaviours that occurred in response to a provocation, frustration, or perceived threat which is best described by frustration-aggression theory (Polman et al., 2007). It is the retaliatory form of aggression motivated by anger (Hubbard et al., 2010; Polman et al., 2007). In contrast, proactive aggression is the cold-blooded and goal-oriented form of aggression (Dodge, 1991; Hubbard et al., 2010; Raine et al., 2006). This instrumental kind of aggression is best conceptualized by the social learning theory that emphasizes the role of operant conditioning and vicarious learning (Bandura, 1973).

Although there is a controversial debate on the usefulness of this distinction (Bushman \& Anderson, 2001; Euler et al., 2017), several studies demonstrated differential correlates of these two subtypes of aggression (Dodge, 1991; Dodge \& Coie, 1987; Hubbard et al., 2010; Kempes et al., 2005; Vitaro et al., 2006), suggesting the different causal pathway of these subtypes. A literature review shows different behavioural and neurocognitive profiles among these groups (Smeets et al., 2017). Proactive aggression is found associated with narcissism (Seah \& Ang, 2008), poor peer relationship in early childhood, blunted affect 
(Raine et al., 2006), higher levels of working memory (Hecht \& Latzman, 2018), and an expectation of positive outcomes (Poulin \& Boivin, 2000). Past researches indicated that correlates of reactive aggression include information processing deficits (Dodge \& Coie, 1987), physical violence(Brendgen et al., 2001), lack of self-control, increased impulsivity, problem-solving deficiency (Atkins et al., 1993), social anxiety, lack of close friend (Raine et al., 2006), narcissistic traits (Bobadilla et al., 2012), increased emotion dysregulation (Hecht \& Latzman, 2018), schizotypal traits, poor interpersonal relations (Seah \& Ang, 2008), social maladjustment, internalizing behavior, and peer rejection (Card \& Little, 2006).

However, there are some inconsistent findings of correlated variables with these two subtypes of aggression. For instance, while in some studies, proactive aggression was associated with delinquency (Atkins et al., 1993; Brendgen et al., 2001; Raine et al., 2006; Vitaro et al., 1998), in some others, it was more related to reactive aggression (Card \& Little, 2006).

The conflicting outcomes might be due to participants' different sex or ages (Bobadilla et al., 2012; Connor et al., 2003; Kempes et al., 2005). Although there are many studies about the development of aggression across the lifespan and comparison of genders, very few accounts for age and gender differences specifically for these subtypes of aggression. About gender comparison, one study showed boys were higher in reactive aggression but no difference in proactive aggression (Kempes et al., 2006; Lickley \& Sebastian, 2018).

Although these subtypes of aggression co-occurrence in children (Merk et al., 2005) and aggression is better conceptualized on a continuous dimension, the distinction between reactive and proactive aggression can still enhance our understanding of their separate precursors and outcomes (Dodge, 1991). This knowledge would contribute to the development of preventive and therapeutic interventions (Hubbard et al., 2010; Merk et al., 2005; Pechorro et al., 2015). As such, reliable and valid measures which could distinguish 
between these two subtypes of aggression are vital. Dodge and Coie (1987) tried to discriminate these two subtypes of aggression among children as young as 3 to 6 years of age. Their six-item rating scale can be completed by teachers, parents, themselves(Kaat et al., 2015), and correctional facility staff (Hubbard et al., 2010). However, self-report measures are privileged over others by accurately recognizing the inherent motivation of aggression, the central feature of this distinction (Raine et al., 2006). The reactive-Proactive Aggression Questionnaire (RPQ) is a brief self-report measure developed by Raine et al. (2006). The psychometric properties of this measure have been investigated by several studies across different cultures, supporting its reliability and validity. (Cenkseven-Önder et al., 2016; Fossati et al., 2009; Fung et al., 2009; Pechorro et al., 2015; Seah \& Ang, 2008). The Cronbach's alpha values in the initial validation of the RPQ among schoolboys aged 7 and 16 years old were 0.84 for the reactive scale, 0.86 for the proactive scale, and 0.90 for the total score (Raine et al., 2006). The Portuguese version of the RPQ showed good psychometric properties, including convergent validity, discriminant validity, and criterion-related validity, as well as internal consistency estimated by Cronbach's alpha and omega coefficient (Pechorro et al., 2018). Cima et al. (2013) have identified Cronbach's alpha of the Dutch version as 0.83 for the reactive scale and 0.87 for the proactive scale, supporting the RPQ as a reliable and valid instrument to identify these subtypes of aggression. Its criterion validity was also established due to the significantly lower scores of non-offenders compared to offenders samples (Cima et al., 2013). The study conducted on Turkish children and adolescents indicated the item-total correlation ranging from 0.40 to 0.74 and an Alpha coefficient above 0.81 for both subscales and the total score, which supports the high internal consistency of this measure (Cenkseven-Önder et al., 2016).

There are studies in favour of the original two-factor model across various cultures. For instance, Fung et al. (2009) have examined the generalizability of the RPQ in an East 
Asian population. Despite the significant correlation between reactive and proactive aggression $(r>0.54)$, the original two-factor model was superior to the one-factor model, and the item- loadings were over 0.45 . A better fit of the two-factor structure was replicated in a study among Portuguese youth (Pechorro et al., 2018). However, the measurement invariance across genders was confirmed after excluding item 21. Although a two-factor model of the Spanish version of the RPQ best fit the data, it was variant across genders and ages, with the best fit in males under 25 years of age (Toro et al., 2020). The original two-factor model of the RPQ also obtained the best fit among Dutch (Cima et al., 2013) and Turkish samples (Cenkseven-Önder et al., 2016).

Despite these findings supporting the two-factor structure of the RPQ, some studies have failed to confirm the original model. Using a person-based approach, Brugman et al. (2017) revealed a three-factor structure based on the severity of aggression among forensic and non-forensic samples. Moreover, the four-factor structure has been explored, combining two subtypes of aggression and various contexts for reactive aggression (playing games and a defensive form) based on a variable-based approach. However, the four-factor solution was considered less solid since items were more uniquely related to each subscale of aggression in the two-factor model of the RPQ. In the same way, Smeets et al. (2017) have extracted three factors in the clinical sample, including reactive aggression due to external provocation, reactive aggression due to internal frustration, and proactive aggression. Pang et al. (2013) also identified three distinct clusters (including high reactive and low proactive aggression, low on both subscales, and high on both subscales) among adolescents in Singapore, providing evidence for this distinction.

Therefore, the contradictory results of the prior researches, together with the lack of study for convergent and criterion validity of the RPQ among the Iranian population, suggest the need for further research on the reliability of the RPQ to distinguish reactive from 
proactive aggression. Hence, this research aims to investigate the replication of the original model of the RPQ (Raine et al., 2006) and its psychometric properties among Iranian adolescents across genders. Thus, we want to examine to what extent these two forms of aggression can differ in a different culture by testing the cross-cultural generalizability of these two kinds of aggression. Therefore, the first hypothesis was that the original two-factor model of the RPQ would also achieve adequate fit while the loadings of items would have a similar pattern across genders. Secondly, the RPQ is predicted to show good internal consistency as measured by the Omega and Alpha coefficient, mean inter-item correlation (MIIC), and corrected item-total correlation in both groups. Thirdly, we hypothesized that both RPQ subscales would relate positively to criterion-related variables, including rulebreaking behaviour, aggressive behaviour, and conduct problems, in a similar way in both groups. Finally, to support that proactive aggression is associated with delinquency (Bobadilla et al., 2012), we examine whether detained individuals can be distinguished based on their scores in the proactive aggression subscale. It was predicted incarcerated individuals would score higher than non-incarcerated adolescents on the proactive subscale.

\section{Method}

\section{Participants}

A total of 450 students participated in this study $\left(\mathrm{M}_{\mathrm{age}}=14\right.$ years; S.D. =.81) consist of 217 boys $\left(\mathrm{M}_{\mathrm{age}}=14.29\right.$ years; $\left.\mathrm{SD}=.80\right)$ and 233 girls $\left(\mathrm{M}_{\mathrm{age}}=13.89\right.$ years; $\left.\mathrm{SD}=.78\right)$. There were 133 students from grade 7, 146 students from grade 8, and 171 students from grade 9. The participants were recruited via a stratified sampling method from 6 secondary schools in Yazd, in the middle of Iran, considering various socioeconomic statuses.

A subsample of 145 students including 69 girls $\left(\mathrm{M}_{\mathrm{age}}=13.6\right.$ years; $\left.\mathrm{SD}=.93\right)$ and 76 boys $\left(\mathrm{M}_{\mathrm{age}}=13.8\right.$ years; $\left.\mathrm{SD}=1.05\right)$ were used to measure convergent validity. This sample 
comprised of the minimum participants needed $(\mathrm{N}=67)$ to achieve the desired statistical power (i.e., 0.80) recommended by Cohen (1988) with the medium effect size (i.e., $r=0.30$ ).

The RPQ was administered in a forensic context to establish criterion validity. Since there was no girl in imprisonment, all the incarcerated boys $(\mathrm{N}=31)$ aged 15 to $19\left(\mathrm{M}_{\mathrm{age}}=\right.$ 17.4 years; $\mathrm{SD}=1.01$ ) in the juvenile detention centre of Yazd were recruited. This sample size was enough to achieve an acceptable level of statistical power (Cohen, 1988) with a medium effect size (i.e., $r=0.50$ ).

\section{Measure}

\section{Reactive-Proactive aggression questionnaire (RPQ)}

Reactive-Proactive aggression questionnaire: Participants completed RPQ, a 23 items questionnaire that is suitable for a wide age range and evaluates two functions of physically and verbally aggressive behaviours (Raine et al., 2006). There are 11 items in the proactive and 12 items in the reactive subscale. Each item is rated on a three-point scale scored with 0 (Never), 1 (Sometimes), and 2 (Often).

\section{Child Behaviour Checklist (CBCL)}

The emotional and behavioural problems were assessed by the Youth Self-Report (YSR) version of the child behaviour checklist for children aged 6-18 (Achenbach \& Rescorla, 2014). This report measure contains 113 items rated on a three-point scale $(0=$ Absent, $1=$ Occurs sometimes, $2=$ Occurs often). It includes subscales that assess anxiety, depression, somatic complaints, social problems, thought problems, attention problems, rulebreaking behaviour and aggressive behaviour. Symptoms of Rule-Breaking Behaviour and Aggressive Behaviour make externalizing problems score. There is excellent support for the psychometric properties of CBCL among the Iranian population and different countries (Achenbach \& Rescorla, 2014). In this study, two subscales of aggressive and rule-breaking behaviours were used to evaluate convergent validity. 


\section{Strengths and Difficulties Questionnaire (SDQ)}

A self-report version of the SDQ (Goodman et al., 1998) measured emotional and behavioural disorders. It comprises 25 items assessing positive and negative attributes of children and adolescents across five subscales: Conduct Problems, Inattention- Hyperactivity, Emotional Symptoms, Peer Problems, and Prosocial Behaviour. Items are rated on a threepoint Likert-type scale with 'not true' (scored 0), 'somewhat true' (achieved 1), and 'certainly true (scored 2). The Persian version of SDQ demonstrated high validity and reliability in several studies (Tehranidoust et al., 2007). In this study, only the conduct problem subscale $(\alpha=0.72)$ was used.

\section{Procedure}

Permission is asked from the developer of the original RPQ before the initial questionnaire was translated and back-translated into Persian. Face and content validity are assessed by two experts, causing a minor change in the phraseology. After the approval of the study in the ethical board of the department of education, three schools were chosen by chance from each educational region. Randomly selected participants completed the questionnaire after their consent got obtained. The questionnaire was accomplished in a group following that the written instruction was read to assure understanding. All subjects were informed that they could withdraw at any time without negative consequences for them. They were encouraged to ask any questions about understanding the content.

Permission was obtained from the state prisons organization to attend the juvenile detention centre of Yazd. The same procedures were done for incarcerated boys, except that questionnaire was read for five illiterate participants. Data were analysed using IBM SPSS Statistics and SPSS Amos version 23. 


\section{Statistical analyses}

Confirmatory factor analysis with maximum-likelihood estimation was done to examine the factor structure of the RPQ. We used Bollen- Stine bootstrapping due to the nonnormality of items at the multivariate level. Considering recommended setting at 5000 samples in bootstrapping analysis (Hair Jr et al., 2014), the bias-corrected confidence interval at the $95 \%$ confidence level was computed based on 5000 bootstrap samples. Model fit of different factor structures suggested for this scale were assessed. Therefore, the original twofactor model has been compared with the one-factor, second-order, and three-factor models (Smeets et al., 2017). All items are loaded on one latent factor named general aggression in the one-factor model, while the factor variance was constrained at 1.00. Different Goodness of fit indices were used for the investigation of model fit, including Comparative Fit Index (CFI), Tucker-Lewis fit index (TLI), the Goodness of its index (GFI), and the Root Mean Square Error of Approximation (RMSEA). Besides, model chi-square divided by degrees of freedom $(\chi 2 / d f)$ is also considered in a way that values less than 2 and 5 are held to be good and acceptable, respectively (Schumacker \& Lomax, 2004). The acceptable cutoff value for CFI is 0.90, indicating adequate fit (Hoyle, 1995). Hu and Bentler (1999) recommended that CFI >.95, TLI $\geq .95$ and RMSEA <.06 indicate a good-fitting model. Akaike information criterion (AIC), Bayesian information criterion (BIC) and expected cross-validation index (ECVI) were used for non-nested models' comparison in the way that the lowest value indicates better fit (Brown, 2015; Kline, 2015). Since factor loadings are considered meaningful when they are above .30 (Pechorro et al., 2018), items with standardized loading lower than that value were removed. No modification indexes (i.e., estimates resulting from validity assessment of constrained parameters in the model) were used to improve the model fit. Then measurement invariance of the primary model was assessed, using multigroup CFA (Milfont \& Fischer, 2010). This approach addresses whether there are differences in the 
measurement of latent constructs across various groups. At first, the configural model is defined in which all parameters were allowed to be freely estimated across groups. This model is the baseline for comparison and estimates the equivalence of the overall factor structure in groups. At the next step, metric invariance would be assessed to investigate whether the same observations indicate the same latent constructs across different groups. Therefore, while item intercepts are still allowed to be freely estimated, the factor loadings are constrained to be equivalent across groups. At the final step, scalar invariance examines the similarity of item intercepts across groups. Support for scalar invariance allows us for comparison of factor means between groups(Lee, 2018). In each step, the baseline model is compared with the constrained model. The difference between these nested models is assessed based on the significance of the chi-square difference test as well as $\Delta \mathrm{CFI}$ and the $\triangle$ RMSEA (Tracey \& Xu, 2017). Therefore, a significant deterioration in the fitness of the more constrained model alongside $\Delta \mathrm{CFI}>0.01$ and $\triangle \mathrm{RMSEA}>0.015$ are considered evidence of non-invariance (Cheung \& Rensvold, 2002).

In the last step, the psychometric properties of the two-factor model were investigated across genders. Internal consistency was assessed using Omega and Alpha coefficients, mean inter-item correlation (MIIC) and corrected item-total correlations (CITC). Omega and Alpha values $\geq .70$, the minimum mean inter-item correlations within the range of .15 to .20 and corrected item-total correlations above .20 were considered adequate internal consistency (Clark \& Watson, 2016; Hair, 2009; Pechorro et al., 2018). Convergent validity was examined using correlation analysis between the residual scores of reactive and proactive aggression and conduct problem, aggressive and rule-breaking behaviours. Criterion validity was examined by comparing the reactive and proactive scores between individuals with and without delinquency. 


\section{Results}

\section{Factor structure and invariance assessment}

As can be seen in Table 1, all models showed better fit to data compared to the null model. However, the two-factor model $\left(\chi^{2} / \mathrm{df}=1.92 ; \mathrm{TLI}=.86 ; \mathrm{CFI}=.88 ; \mathrm{RMSEA}=.04\right)$ fit the data better than the one-factor model $\left(\chi^{2} / \mathrm{df}=2.08 ; \mathrm{TLI}=.82 ; \mathrm{CFI}=.84 ; \mathrm{RMSEA}=\right.$ $.05)$, second-factor model $(\chi 2 / \mathrm{df}=2.86$; TLI $=.70 ; \mathrm{CFI}=.72$; RMSEA $=.06)$, and threefactor model $(\chi 2 / \mathrm{df}=1.87 ; \mathrm{TLI}=.86 ; \mathrm{CFI}=.87 ; \mathrm{RMSEA}=.05)$, considering the lowest amount of AIC, BIC and ECVI. All item loadings exceed the recommended value of 0.30 (Pechorro et al., 2018), except for item 15.

In the next stage, two groups were defined based on gender to evaluate the measurement invariance of the RPQ and the impact of gender on the model fit. The result of multiple group CFA presented in Table 2 indicated that loadings of items have a different pattern across genders (Tracey \& Xu, 2017). The comparison of the baseline model with the metric model revealed non-equality of factor loading across genders. As shown in Table 2, although $\triangle$ RMSEA was not more than $0.015, \triangle \mathrm{CFI}$ exceeded 0.01 showing weak invariance was not established (Cheung \& Rensvold, 2002). Moreover, the chi-square difference test $(\Delta \chi 2=161.59, \mathrm{df}=23, \mathrm{P}<.05)$ showed a significantly poorer fit for the model with constrained factor loadings across groups. Further assessment was not justified because the least strict model was not confirmed to be equal. (Lee, 2018; Putnick \& Bornstein, 2016).

Factor loadings of items were examined across genders to explore the exact dissimilarity of model form between groups. The result indicated different items with factor loading less than .30 across genders (items 4, 15, 18, 21 among girls and 13 and 15 among boys). The result of confirmatory factor analysis revealed a better fit of the two-factor structure of the RPQ after removing items with factor loadings less than the threshold compared to the various models proposed for the RPQ in both groups (Table 3). Therefore, 
the two-factor intercorrelated structures were considered the best representation of the RPQ, considering reasonable fit and numerous researches supporting evidence for this model. The standardized item loadings for the two-factor model for each group and the total sample are presented in Table 4.

\section{Internal consistency}

The omega coefficient for RPQ total score, reactive and proactive subscales among girls was almost $.84, .78$ and .68 , respectively. In the other group, omega coefficient was as follow: total score $=.85$, reactive subscale $=.74$ and proactive subscale $=.77$. The result of internal consistency for the RPQ subscales, estimated by Cronbach's alpha, can be considered acceptable except for the proactive subscale among girls (Table 5).

CITC for reactive aggression ranged .33 to .45 for boys $(\mathrm{M}=7.86, \mathrm{SD}=3.50)$ and .33 to .55 for girls $(\mathrm{M}=8.76, \mathrm{SD}=3.94)$. For proactive aggression, $\mathrm{CITC}$ ranged .30 to .44 for girls $(\mathrm{M}=2.03, \mathrm{SD}=2.07)$ and .32 to .56 for boys $(\mathrm{M}=3.47, \mathrm{SD}=3.24)$. Reactive and proactive aggression scores were significantly correlated among girls $(\mathrm{r}=.61, \mathrm{p}<001)$ and boys $(\mathrm{r}=.63 \mathrm{p}<001)$.

\section{Convergent validity}

In a subsample of 145 students (76 males and 69 females), convergent validity was assessed separately for each gender, evaluating the relationship between one subscale of SDQ (conduct problem) and two subscales of CBCL (aggressive and rule-breaking behaviours) and the two RPQ subscales. Once more, we rerun the correlation analysis with residuals of reactive and proactive aggression scores, considering the substantial correlation between the two types of aggression.

In males, standardized residuals of reactive aggression were significantly correlated with rule-breaking $(\mathrm{r}=.26, \mathrm{p}<.05)$ and aggressive behaviours subscales of CBCL $(\mathrm{r}=.36, \mathrm{p}<$ .01). Aggressive behaviours subscales of CBCL also significantly correlated with the residual 
of reactive aggression scores among girls $(\mathrm{r}=.30, \mathrm{p}<.05)$. While purely proactive aggression did not show any significant correlation among boys, it was significantly associated with rule-breaking $(r=28, \mathrm{p}<.05)$ and aggressive behaviours scores $(\mathrm{r}=24, \mathrm{p}<.05)$ in the other group. Besides, the result revealed that the raw score of both factors and the RPQ total score were significantly correlated with other variables in a positive direction (Table 6). A marginally significant correlation between residual reactive aggression and conduct problem scores $(\mathrm{r}=22, \mathrm{p}=.06)$ was seen among girls. In both groups, the strongest correlations were between the raw score of reactive aggression and aggressive behaviours (Male: $r=.64$, Female: $\mathrm{r}=.54, \mathrm{p}<.001)$.

\section{criterion validity}

An independent-samples t-test was conducted to compare the aggression scores of 50 students aged 15 with a sample of incarcerated boys $(\mathrm{N}=31)$. As shown in table 7 , there was no significant difference in reactive aggression scores between incarcerated individuals $(\mathrm{M}=8.93, \mathrm{SD}=3.66)$ and non-incarcerated sample $(\mathrm{M}=7.86, \mathrm{SD}=3.50)$; $\mathrm{t}(79)=1.32, \mathrm{p}=.19)$. But result demonstrated a significant effect for grouping in proactive aggression scores, $t(79)$ $=2.32, \mathrm{p}=02$. , showing incarcerated boys obtained higher scores $(\mathrm{M}=4.49, \mathrm{SD}=.80)$ than the other group $(\mathrm{M}=3.12, \mathrm{SD}=.44)$. Hedges' $\mathrm{g}$ was calculated $(\mathrm{g}=0.94)$ to assess the effect size due to the different sample sizes of the compared groups (Lakens, 2013).

\section{Discussion}

This study investigated the factor structure and psychometric properties of reactive and proactive aggression questionnaires across genders among Iranian adolescents. Overall, the result confirmed the cross-cultural stability of two distinct types of aggression, representing a better fit of the two-factor intercorrelated model than the other proposed structures. 
This result is consistent with research confirming this model's generalizability among various cultures (Cima et al., 2013; Fung et al., 2009; Goodman et al., 1998; Raine et al., 2006). However, there are studies indicating different factor structures (Brugman et al., 2017; Colins, 2016; Pang et al., 2013; Smeets et al., 2017). This discrepancy might be justified by the variety of attitudes in various cultures, causing different expressions of aggression. Besides, findings could be influenced by the different samples among these studies. Therefore, these subtypes of aggression could be more distinguishable in non-clinical samples (Smeets et al., 2017).

Although the CFA revealed that the two-factor model had a better fit than different suggested models, the fit indices did not reach the acceptable cut off value. However, similar results in a study by Toro et al. (2020) showed better model fit indicators after executing residual covariances. In this study, similarly, the modification indexes suggested covariances of the measurement error among the items of the same factor; however, no post-hoc alteration to the model was done to prevent data-driven changes.

The model's poor fit can also be rooted in the wording and ambiguity of phrases so that some items can be interpreted as both reactive and proactive. This idea is also confirmed by various studies reporting the cross-loading of several items of RPQ (Brugman et al., 2017; Fossati et al., 2009). The result of an explorative factor analysis conducted among adults, which revealed that only a subset of items $(1,5,7,11$, and 14 for reactive and items $2,6,9$, 10,12 , and 20 for proactive aggression) could differentiate adequately between these two subscales (Lobbestael et al., 2013). Moreover, this scale examines aggressive behaviours coupled with aggressive feelings. For example, in the reactive subscale, items 5,11,13,14 and somehow 22 tended to examine the feeling of aggression more. It is also suggested that these two forms of aggression would be better distinguished with behavioural observations and the questionnaires disentangling form and function (Polman et al., 2007) or based on the target of 
aggression (whether person or object). Future studies should consider the association between such measures and the RPQ factor structure. Furthermore, this result can lend support for the dimensional compared to the categorical approach due to the fuzzy distinction between these two types of aggression (Dodge, 1991) and their considerable corelation. Besides, proactive aggression could be displayed with a delay from prior provocation (Merk et al., 2005), making distinguishing these subtypes based on a questionnaire more complicated.

In this paper, the measurement invariance of the RPQ was examined across genders. In line with studies indicating variants of the RPQ across genders among different populations (Rodríguez et al., 2009; Toro et al., 2020), the result of CFA revealed two distinctive patterns of item loading across genders. Hence, the comparison of aggression between Iranian boys and girls based on this measure is not justified. Moreover, the result did not support replicating the factor structure presented by Raine et al. (2006) across genders. There was no making obscene phone calls, carrying weapons, and taking things from other students as proactive aggression in girls. But, boys did not show madness and anger after losing in games as reactive aggression. In addition, it seems the trait of proactive aggression among Iranian adolescents did not include using force to obtain money, considering poor factor loading of item 15 in both groups. These findings are comparable with studies indicating measurement invariance of the RPQ after modification of the model. For example, a study by Baker et al. (2008) reported the invariance of the RPQ across genders after implementing certain constraints (e.g., letting for correlation of error variance). Structural invariance of the RPQ was also confirmed among Portuguese youth after excluding item 21 (Pechorro et al., 2018).

The impact of gender on the expression of aggression alongside cultural issues should be considered to interpret these findings. Additionally, because few studies investigated the measurement invariance of the RPQ across genders, it is suggested to ascertain the variant of 
this scale by further researches. Moreover, keeping in mind that there were different ages in each group in this research, further study on the invariance assessment of this scale would benefit from more homogeneous samples.

Another purpose of this study was to examine the reliability and validity of the RPQ across genders. Omega and Alpha coefficients indicated sufficient internal consistency of the RPQ (Deng \& Chan, 2017; Nunnally, 1994), except for the proactive subscale in females. Because the inter-relatedness of the items in each subscale was not poor, heterogeneity in this subscale is not the case (Tavakol \& Dennick, 2011). However, further investigation is needed to examine whether a low omega coefficient might result from the reduction in the items of the proactive subscale (Tavakol \& Dennick, 2011) or the inability of this questionnaire to measure proactive aggression in girls (Brugman et al., 2017).

The total score of the RPQ, like previous studies, was correlated to subscales of CBCL and SDQ in both groups, supporting convergent validity of the RPQ (Bartels et al., 2018). In line with previous studies (Fossati et al., 2009; Raine et al., 2006), residual scores were used to take into account the variance shared with the other aggressive dimension due to a moderate correlation between both subscales. When the residual scores were used, rulebreaking behaviours were significantly associated with purely proactive aggression among girls. This result is consistent with the notion that this kind of aggression is more instrumental and planned (Fossati et al., 2009). In contrast, the rule-breaking score was correlated with a purely reactive score among boys, suggesting that boys who demonstrate aggression followed by provocation tend to engage in delinquency and violate rules.

Furthermore, the aggressive behaviour subscale of CBCL showed a moderate correlation with purely reactive aggression in both groups, suggesting reactive aggression is a predominant form of aggression (Thomson \& Centifanti, 2018). However, the association between aggressive behaviours and purely proactive aggression was significant only among 
girls, confirming distinctive patterns of association across genders (Connor et al., 2003). This result implied that girls with high aggressive behaviours display both profiles of aggression which is consistent with researches indicating the mixture of both kinds of aggression among the more aggressive individuals (Colins, 2016; Euler et al., 2017; Fossati et al., 2009; Merk et al., 2005).

Since residual proactive aggression did not show any significant correlation among boys, further investigation is needed to figure out the reason behind these results. There might be some issues with the male's proactive subscale. On the other hand, using the residual score to control for the effect of the other dimension may contribute to these results due to increasing error variance (Raine et al., 2006). Besides, keeping in mind that these correlates are in common between reactive and proactive aggression, subsequent studies should notice more specific variables to support more evidence that each type of aggression comes in combination with differential correlates. Overall, our hypothesis regarding convergent validity of the RPQ scale was supported while the correlates of the RPQ subscales differed partly across genders. Therefore, this paper provides more evidence for reactive and proactive aggression independence in line with previous researches (Connor, 2004).

Data on this study support the studies suggesting that proactive aggression is related to delinquency and antisocial behaviours (Brendgen et al., 2001; Deng \& Chan, 2017), as incarcerated boys showed a significantly higher level of proactive aggression but not reactive form. This result contrasts with the study showing the association between delinquency and the severity of aggression but not the types (Stickle et al., 2012). However, this result lends support for the association between severe aggression, psychopathic and callous-unemotional traits and proactive but not reactive aggression (Brugman et al., 2017; Cima \& Raine, 2009; Cima et al., 2013). This result signifies differential trajectories for these two kinds of aggression (Poulin \& Boivin, 2000; Raine et al., 2006). However, the non-significance result 
of mean difference of reactive aggression between normal and incarcerated groups is consistent with the fact that a moderate level of reactive aggression is normative (Brugman et al., 2017; Fossati et al., 2009; Raine et al., 2006), suggesting reactive aggression as a relatively common type (Cima et al., 2013; Fung et al., 2009).

Furthermore, this study can provide evidence for the ability of this scale to screen adolescents with proactive aggression, despite a disagreement on the existence of a solely proactive aggressive population (Merk et al., 2005; Smeets et al., 2017) or the ability of the RPQ in recognizing such group (Brugman et al., 2017). However, this result contrasts with research reporting significantly higher reactive and proactive aggression scores in violent offenders than non-offender (Cima et al., 2013). This discrepancy could be explained through disparate types of crimes in our study, including violent and non-violent together.

The current study confronts some limitations. This study is the first to examine the psychometric properties of the RPQ while comparing the RPQ factor structure across two genders in Iranian populations. Since there is strong evidence for validation of the theoretical factor structure of the RPQ across different cultures, the result of the current study should be considered with care. More study is needed to examine whether this result is affected by the data's artefact or actual cultural context differences. Therefore, the results in this regard should be interpreted cautiously. This cross-sectional study could not present the differential pathways of these two types of aggression as well as the temporal stability of the RPQ. A future longitudinal study is encouraged to measure the invariance of factor structure across time. Since this study used one source of information for all assessments, future examination for the evaluation of criterion validity of the RPQ should consider observational assessment and multiple informants like friends, parents and teachers, as well as computational instruments. In this study, the psychometric properties of the RPQ were confirmed among non-clinical adolescents. We suggest replicating this study with a higher risk sample to 
evaluate the usefulness of this brief and easy answer questionnaire for the clinical population, considering the co-occurrence of these two types of aggression among high aggressive individuals (Merk et al., 2005). Therefore, regarding the moderate to a strong correlation between these subtypes of aggression (Fossati et al., 2009; Hubbard et al., 2010; Poulin \& Boivin, 2000; Raine et al., 2006) and concerns about the usefulness of this distinction, repetition of this study among cases with the extreme score is suggested.

Since there was not any female incarcerated, the generalizability of the result in this regard is limited. Regarding a severity model of reactive and proactive aggression against a typology model (Stickle et al., 2012), future researches should take into account the severity of aggression and psychiatric diagnosis in the relationship between types of aggression and delinquency in a larger sample including both genders (Cima et al., 2013). Such studies would provide the possibility to compare the exclusive role of reactive and proactive aggression in various types of crimes across genders.

Altogether, this study adds to the knowledge of two distinct forms of aggression among Iranian adolescents. Data in this study did not support the invariance of the original factor structure of the RPQ across genders, implying boys and girls express reactive and proactive aggression differently. However, the two-factor structure represented the data as a reasonable well-fitting model with adequate reliability and validity across genders. This study signifies future investigation of the distinctive correlation between these two forms of aggression and emotional and cognitive deficits across both genders and ages. In this regard, scales dissociating different facets of aggression alongside functions are recommended to configure better the distinctive correlates of reactive and proactive aggression. Such studies would confirm unique etiological and consequential pathways and shed light on producing more efficient and specific intervention and prevention strategies through attention to specific underlying mechanisms. 


\section{References}

Atkins, M. S., Stoff, D. M., Osborne, M. L., \& Brown, K. (1993). Distinguishing instrumental and hostile aggression: does it make a difference? Journal of abnormal child psychology, 21(4), 355-365.

Baker, L. A., Raine, A., Liu, J., \& Jacobson, K. C. (2008). Differential genetic and environmental influences on reactive and proactive aggression in children. Journal of abnormal child psychology, 36(8), 1265-1278.

Bandura, A. (1973). Aggression: A social learning analysis. prentice-hall.

Bartels, M., Hendriks, A., Mauri, M., Krapohl, E., Whipp, A., Bolhuis, K., Conde, L. C., Luningham, J., Ip, H. F., \& Hagenbeek, F. (2018). Childhood aggression and the cooccurrence of behavioural and emotional problems: results across ages 3-16 years from multiple raters in six cohorts in the EU-ACTION project. European child \& adolescent psychiatry, 27(9), 1105-1121.

Bobadilla, L., Wampler, M., \& Taylor, J. (2012). Proactive and reactive aggression are associated with different physiological and personality profiles. Journal of Social and clinical psychology, 31(5), 458-487.

Brendgen, M., Vitaro, F., Tremblay, R. E., \& Lavoie, F. (2001). Reactive and proactive aggression: Predictions to physical violence in different contexts and moderating effects of parental monitoring and caregiving behavior. Journal of abnormal child psychology, 29(4), 293-304.

Brown, T. A. (2015). Confirmatory factor analysis for applied research. Guilford publications.

Brugman, S., Cornet, L. J., Smeijers, D., Smeets, K., Oostermeijer, S., Buitelaar, J. K., Verkes, R. J., Lobbestael, J., de Kogel, C. H., \& Jansen, L. M. (2017). Examining the reactive proactive questionnaire in adults in forensic and non-forensic settings: A variable-and person-based approach. Aggressive Behavior, 43(2), 155-162.

Bushman, B. J., \& Anderson, C. A. (2001). Is it time to pull the plug on hostile versus instrumental aggression dichotomy? Psychological review, 108(1), 273.

Card, N. A., \& Little, T. D. (2006). Proactive and reactive aggression in childhood and adolescence: A meta-analysis of differential relations with psychosocial adjustment. International journal of behavioral development, 30(5), 466-480.

Cenkseven-Önder, F., Avci, R., \& Çolakkadioglu, O. (2016). Validity and Reliability of the Reactive-Proactive Aggression Questionnaire in Turkish Adolescents. Educational Research and Reviews, 11(20), 1931-1943.

Cheung, G. W., \& Rensvold, R. B. (2002). Evaluating goodness-of-fit indexes for testing measurement invariance. Structural equation modeling, 9(2), 233-255.

Cima, M., \& Raine, A. (2009). Distinct characteristics of psychopathy relate to different subtypes of aggression. Personality and individual differences, 47(8), 835-840.

Cima, M., Raine, A., Meesters, C., \& Popma, A. (2013). Validation of the Dutch Reactive Proactive Questionnaire (RPQ): Differential correlates of reactive and proactive aggression from childhood to adulthood. Aggressive Behavior, 39(2), 99-113.

Clark, L. A., \& Watson, D. (2016). Constructing validity: Basic issues in objective scale development.

Cohen, J. (1988). Statistical power analysis for the behavioral sciences [Internet]. Statistical Power Analysis for the Behavioral Sciences, 567. 
Colins, O. F. (2016). Assessing reactive and proactive aggression in detained adolescents outside of a research context. Child Psychiatry \& Human Development, 47(1), 159172.

Conaty, J. (2006). The correlates of proactive and reactive aggression in early childhood. The University of North Carolina at Greensboro.

Connor, D. F. (2004). Aggression and antisocial behavior in children and adolescents: Research and treatment. Guilford Press.

Connor, D. F., Steingard, R. J., Anderson, J. J., \& Melloni, R. H. (2003). Gender differences in reactive and proactive aggression. Child psychiatry and human development, 33(4), 279-294.

Deng, L., \& Chan, W. (2017). Testing the difference between reliability coefficients alpha and omega. Educational and psychological measurement, 77(2), 185-203.

Dodge, K. A. (1991). The structure and function of reactive and proactive aggression. The development and treatment of childhood aggression, 16(5), 201-218.

Dodge, K. A., \& Coie, J. D. (1987). Social-information-processing factors in reactive and proactive aggression in children's peer groups. Journal of personality and social psychology, 53(6), 1146.

Euler, F., Steinlin, C., \& Stadler, C. (2017). Distinct profiles of reactive and proactive aggression in adolescents: Associations with cognitive and affective empathy. Child and adolescent psychiatry and mental health, 11(1), 1-14.

Fossati, A., Raine, A., Borroni, S., Bizzozero, A., Volpi, E., Santalucia, I., \& Maffei, C. (2009). A cross-cultural study of the psychometric properties of the ReactiveProactive Aggression Questionnaire among Italian nonclinical adolescents. Psychological assessment, 21(1), 131.

Fung, A. L.-C., Raine, A., \& Gao, Y. (2009). Cross-cultural generalizability of the reactiveproactive aggression questionnaire (RPQ). Journal of personality assessment, 91(5), 473-479.

Goodman, R., Meltzer, H., \& Bailey, V. (1998). The Strengths and Difficulties Questionnaire: A pilot study on the validity of the self-report version. European child \& adolescent psychiatry, 7(3), 125-130.

Hair, J. F. (2009). Multivariate data analysis.

Hair Jr, J. F., Sarstedt, M., Hopkins, L., \& Kuppelwieser, V. G. (2014). Partial least squares structural equation modeling (PLS-SEM): An emerging tool in business research. European business review.

Hecht, L. K., \& Latzman, R. D. (2018). Exploring the differential associations between components of executive functioning and reactive and proactive aggression. Journal of clinical and experimental neuropsychology, 40(1), 62-74.

Hoyle, R. H. (1995). Structural equation modeling: Concepts, issues, and applications. Sage.

Hu, L. t., \& Bentler, P. M. (1999). Cutoff criteria for fit indexes in covariance structure analysis: Conventional criteria versus new alternatives. Structural equation modeling: a multidisciplinary journal, 6(1), 1-55.

Hubbard, J. A., McAuliffe, M. D., Morrow, M. T., \& Romano, L. J. (2010). Reactive and proactive aggression in childhood and adolescence: Precursors, outcomes, processes, experiences, and measurement. Journal of personality, 78(1), 95-118.

Justice, N. C. f. J., \& Justice, U. S. O. o. J. (1999). Juvenile Offenders and Victims: National Report. National Center for Juvenile Justice.

Kaat, A. J., Farmer, C. A., Gadow, K. D., Findling, R. L., Bukstein, O. G., Arnold, L. E., Bangalore, S. S., McNamara, N. K., \& Aman, M. G. (2015). Factor validity of a proactive and reactive aggression rating scale. Journal of child and family studies, 24(9), 2734-2744. 
Kempes, M., Matthys, W., De Vries, H., \& Van Engeland, H. (2005). Reactive and proactive aggression in children A review of theory, findings and the relevance for child and adolescent psychiatry. European child \& adolescent psychiatry, 14(1), 11-19.

Kempes, M., Matthys, W., Maassen, G., van Goozen, S., \& van Engeland, H. (2006). A parent questionnaire for distinguishing between reactive and proactive aggression in children. European child \& adolescent psychiatry, 15(1), 38-45.

Kline, R. B. (2015). Principles and practice of structural equation modeling. Guilford publications.

Lakens, D. (2013). Calculating and reporting effect sizes to facilitate cumulative science: a practical primer for t-tests and ANOVAs. Frontiers in psychology, 4, 863.

Lee, S. T. (2018). Testing for Measurement Invariance: Does your measure mean the same thing for different participants? APS Observer, 31(8).

Lickley, R. A., \& Sebastian, C. L. (2018). The neural basis of reactive aggression and its development in adolescence. Psychology, Crime \& Law, 24(3), 313-333.

Lobbestael, J., Cima, M., \& Arntz, A. (2013). The relationship between adult reactive and proactive aggression, hostile interpretation bias, and antisocial personality disorder. Journal of personality disorders, 27(1), 53-66.

Merk, W., Orobio de Castro, B., Koops, W., \& Matthys, W. (2005). The distinction between reactive and proactive aggression: Utility for theory, diagnosis and treatment? European Journal of Developmental Psychology, 2(2), 197-220.

Milfont, T. L., \& Fischer, R. (2010). Testing measurement invariance across groups: Applications in cross-cultural research. International Journal of psychological research, 3(1), 111-130.

Nunnally, J. C. (1994). Psychometric theory 3E. Tata McGraw-hill education.

Pang, J. S., Ang, R. P., Kom, D. M., Tan, S. H., \& Chiang, A. Q. (2013). Patterns of Reactive and Proactive Aggression in Young Adolescents in S ingapore. Social Development, 22(4), 794-812.

Pechorro, P., Ayala-Nunes, L., Kahn, R., \& Nunes, C. (2018). The Reactive-Proactive Aggression Questionnaire: Measurement invariance and reliability among a school sample of Portuguese youths. Child Psychiatry \& Human Development, 49(4), 523533.

Pechorro, P., Ray, J. V., Salas-Wright, C. P., Maroco, J., \& Goncalves, R. A. (2015). Adaptation of the Basic Empathy Scale among a Portuguese sample of incarcerated juvenile offenders. Psychology, Crime \& Law, 21(7), 699-714.

Polman, H., de Castro, B. O., Koops, W., van Boxtel, H. W., \& Merk, W. W. (2007). A metaanalysis of the distinction between reactive and proactive aggression in children and adolescents. Journal of abnormal child psychology, 35(4), 522-535.

Poulin, F., \& Boivin, M. (2000). Reactive and proactive aggression: evidence of a two-factor model. Psychological assessment, 12(2), 115.

Putnick, D. L., \& Bornstein, M. H. (2016). Measurement invariance conventions and reporting: The state of the art and future directions for psychological research. Developmental review, 41, 71-90.

Raine, A., Dodge, K., Loeber, R., Gatzke-Kopp, L., Lynam, D., Reynolds, C., StouthamerLoeber, M., \& Liu, J. (2006). The reactive-proactive aggression questionnaire: Differential correlates of reactive and proactive aggression in adolescent boys. Aggressive Behavior: Official Journal of the International Society for Research on Aggression, 32(2), 159-171.

Rodríguez, J. M. A., Fernández, M. E. P., \& Ramírez, J. M. (2009). Cuestionario de agresión reactiva y proactiva: un instrumento de medida de la agresión en adolescentes. Revista de psicopatología y psicología clínica, 14(1), 37-49. 
Schumacker, R. E., \& Lomax, R. G. (2004). A beginner's guide to structural equation modeling. psychology press.

Seah, S. L., \& Ang, R. P. (2008). Differential correlates of reactive and proactive aggression in Asian adolescents: Relations to narcissism, anxiety, schizotypal traits, and peer relations. Aggressive Behavior: Official Journal of the International Society for Research on Aggression, 34(5), 553-562.

Smeets, K., Oostermeijer, S., Lappenschaar, M., Cohn, M., Van der Meer, J., Popma, A., Jansen, L., Rommelse, N., Scheepers, F., \& Buitelaar, J. K. (2017). Are proactive and reactive aggression meaningful distinctions in adolescents? A variable-and personbased approach. Journal of abnormal child psychology, 45(1), 1-14.

Stickle, T. R., Marini, V. A., \& Thomas, J. N. (2012). Gender differences in psychopathic traits, types, and correlates of aggression among adjudicated youth. Journal of abnormal child psychology, 40(4), 513-525.

Tavakol, M., \& Dennick, R. (2011). Making sense of Cronbach's alpha. International journal of medical education, 2, 53.

Tehranidoust, M., Shahrivar, Z., Pakbaz, B., Rezaei, A., \& Ahmadi, F. (2007). Validity of farsi version of strengths and difficulties questionnaire (SDQ). Advances in cognitive science, 8(4), 33-39.

Thomson, N. D., \& Centifanti, L. C. (2018). Proactive and reactive aggression subgroups in typically developing children: The role of executive functioning, psychophysiology, and psychopathy. Child Psychiatry \& Human Development, 49(2), 197-208.

Toro, R. A., García-García, J., \& Zaldívar-Basurto, F. (2020). Factorial Analysis and Invariance Testing for Age and Gender of the Reactive-Proactive Aggression Questionnaire (RPQ). International Journal of Psychological Research, 13(1), 62-70.

Tracey, T. J., \& Xu, H. (2017). Use of multi-group confirmatory factor analysis in examining measurement invariance in counseling psychology research. The European Journal of Counselling Psychology, 6(1), 75-82.

Vitaro, F., Brendgen, M., \& Barker, E. D. (2006). Subtypes of aggressive behaviors: A developmental perspective. International Journal of Behavioral Development, 30(1), 12-19.

Vitaro, F., Gendreau, P. L., Tremblay, R. E., \& Oligny, P. (1998). Reactive and proactive aggression differentially predict later conduct problems. The Journal of Child Psychology and Psychiatry and Allied Disciplines, 39(3), 377-385.

\section{Acknowledgements}

Ethics approval for this project was granted by the Psychology department of Shahid

Beheshti University Ethics Committee. All participants provided informed consent for their participation.

\section{Data availability statement}

Datasets that support the findings of this study can be freely and openly available in Psych Archives at http://dx.doi.org/10.23668/psycharchives.4371 


\section{Table 1}

Model Fitting Results of the RPQ for the Total Sample

\begin{tabular}{lccccccccc}
\hline \multicolumn{1}{c}{ Model } & $\chi^{2}$ & $d f$ & ECVI & BIC & AIC & CFI & GFI & TLI & RMSEA \\
& 1845.90 & 253 & 4.21 & 1986.41 & 1891.90 & & & & \\
\hline Null Model & 480.41 & 230 & 1.27 & 761.43 & 572.41 & .84 & .91 & .82 & .05 \\
One- Factor Model & 663.84 & 232 & 1.67 & 932.64 & 751.84 & .72 & .89 & .70 & .06 \\
Second- Order Model & 425.88 & 227 & 1.16 & 725.23 & 523.88 & .87 & .92 & .86 & .05 \\
Three- Factor Model & 399.72 & 208 & 1.09 & 674.63 & 489.72 & .88 & .93 & .86 & .04 \\
Two- Factor Model & & & & & & & & & \\
\hline
\end{tabular}

Note. All $\chi 2$ values are statistically significant (Bollen-Stine bootstrap $\mathrm{P}=0.001$ ). 
Table 2

The Goodness of Fit Indices for the Assessment of Cross-Gender Invariance of the RPQ

\begin{tabular}{llllllllll}
\hline Model & $\chi 2$ & df & ECVI & AIC & CFI & TLI & RMSEA & $\Delta$ CFI & $\Delta \chi^{2}$ \\
\hline Male & 368.87 & 229 & 2.35 & 508.87 & .84 & .82 & .05 & & \\
Female & 384.72 & 229 & 2.26 & 524.72 & .81 & .79 & .05 & & \\
Configural & 753.60 & 458 & 2.30 & 1033.60 & .83 & .80 & .038 & & \\
Metric & 915.19 & 481 & 2.56 & 1149.19 & .75 & .73 & .045 & .08 & $161.59^{* * *}$ \\
\hline
\end{tabular}


Table 3

Model Fitting Results of the RPQ Across Genders

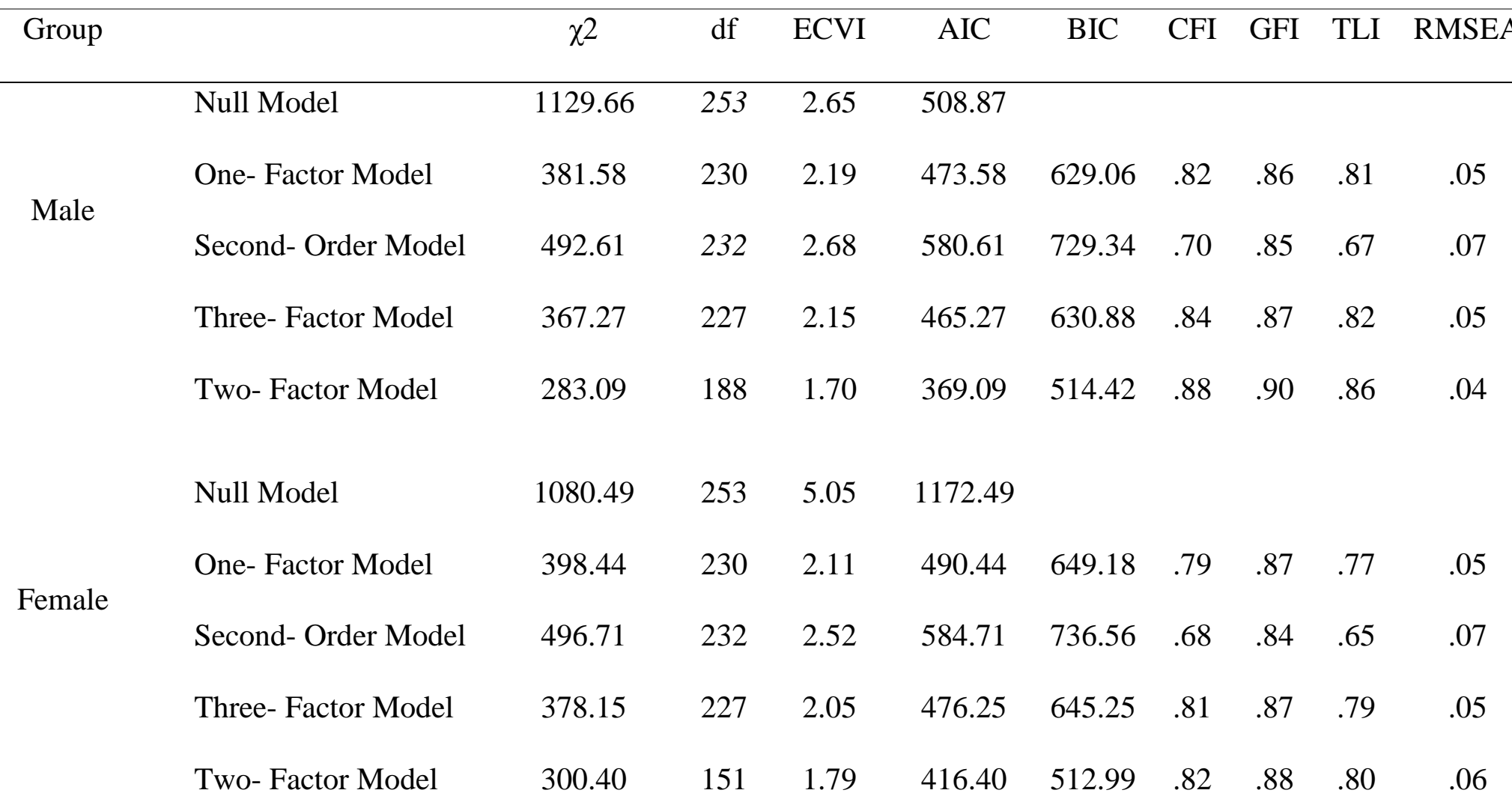


Table 1

$R P Q T$ Two-Factor intercorrelated Structure with Standardized Item Loadings

\begin{tabular}{|c|c|c|c|c|c|c|}
\hline \multirow[t]{2}{*}{ Items } & \multicolumn{3}{|l|}{ Reactive } & \multicolumn{3}{|c|}{ Proactive } \\
\hline & Male & Female & Total & Male & Female & Total \\
\hline 1. Yell when annoyed & .47 & .41 & .44 & & & \\
\hline 3. Angry when provoked & .33 & .36 & .36 & & & \\
\hline 5. Get angry when frustrated & .48 & .37 & .42 & & & \\
\hline 7. Temper tantrum & .51 & .60 & .55 & & & \\
\hline 8. Damage things due to madness & .56 & .47 & .51 & & & \\
\hline 11. Angry when opposed & .43 & .56 & .47 & & & \\
\hline 13. Angry when lose a game & Deleted & .47 & .37 & & & \\
\hline 14. Angry when threatened & .44 & .46 & .47 & & & \\
\hline 16. Feel better after hitting/ yelling & .53 & .53 & .53 & & & \\
\hline 19. Hit others to defend & .42 & .51 & .45 & & & \\
\hline 22. Hit when teased & .50 & .64 & .57 & & & \\
\hline 2. Fight for status & & & & .48 & .47 & .47 \\
\hline 4. Take things & & & & .62 & Deleted & .44 \\
\hline 6. Vandalize for fun & & & & .57 & .50 & .55 \\
\hline 9. Gang fights & & & & .47 & .43 & .48 \\
\hline 10. Hurt to win & & & & .37 & .43 & .42 \\
\hline 12. Manipulate using force & & & & .48 & .39 & .47 \\
\hline 15. Get money by force & & & & Delet & Deleted & Delete \\
\hline
\end{tabular}


17. Threaten and bullying others

.60

.57

.57

18. Obscene phone calls

$.47 \quad$ Deleted .39

20. Gang manipulation

.40

.39

.40

21. Carry weapon

.43

Deleted .36

23. Yell to manipulate

.43

.48

.45 


\section{Table 5}

Internal Consistency for the RPQ across genders

\begin{tabular}{lccccccccc}
\hline & \multicolumn{3}{c}{ Female } & \multicolumn{5}{c}{ Male } \\
\cline { 2 - 9 } & Omega & Alpha & MIIC & CITCR & Omega & Alpha & MIIC & CITCR \\
Reactive & 0.78 & .77 & 0.24 & $0.35-0.55$ & 0.74 & .74 & 0.22 & $0.33-0.45$ \\
proactive & 0.68 & .67 & 0.21 & $0.30-0.44$ & 0.78 & .77 & 0.23 & $0.32-0.56$ \\
Total aggression & 0.83 & .83 & 0.21 & $0.30-0.54$ & 0.85 & .85 & 0.21 & $0.30-0.53$ \\
\hline
\end{tabular}




\section{Table 6}

Convergent Validity of the $R P Q$ across genders

\begin{tabular}{|c|c|c|c|c|c|c|}
\hline Variable & & Purely reactive & Purely proactive & Raw Reactive & Raw Proactive & Total Score \\
\hline Rule breaking & & .14 & $.28 *$ & $.35 * *$ & $.42 * *$ & $.43 * * *$ \\
\hline $\begin{array}{l}\text { Aggressive } \\
\text { behavior }\end{array}$ & Female & $.30 *$ & $.24^{*}$ & $.50 * *$ & $.47 * *$ & $.55^{* * *}$ \\
\hline Conduct disorder & & .22 & .14 & $.35^{* *}$ & $.30 *$ & $.37 * *$ \\
\hline Rule breaking & & $.26^{*}$ & .19 & $.56^{* *}$ & $.53 * *$ & $.59 * *$ \\
\hline $\begin{array}{l}\text { Aggressive } \\
\text { behavior }\end{array}$ & Male & $.36 * *$ & .12 & $.64 * *$ & $.54 * *$ & $.64 * *$ \\
\hline Conduct disorder & & .10 & .15 & $.30 * *$ & $.32 * *$ & $.33^{* *}$ \\
\hline
\end{tabular}

Note. $* \mathrm{p}<.05, * * \mathrm{p}<.01, * * * \mathrm{p}<.001$ 


\section{Table 7}

Independent Samples T-Test for Comparison of the Incarcerated Boys and NonIncarcerated Adolescents on the RPQ Scores

95\% CI for Cohen's d

\begin{tabular}{|c|c|c|c|c|c|}
\hline & $\mathrm{t}$ & $\mathrm{df}$ & $\mathrm{p}$ & Lower & Upper \\
\hline Reactive & 1.32 & 79 & 0.19 & -0.54 & 2.69 \\
\hline Proactive & 2.32 & 79 & 0.02 & 0.28 & 3.65 \\
\hline
\end{tabular}

